Cuestiones de filosofía

ISSN: 0123-5095

Vol. 1 - No. 17

Año 2015

pp. $275-294$

\title{
DE PÁJAROS, MOHOS Y ÁTOMOS: HACIA UNA NOCIÓN POSHUMANA DE EXPRESIVIDAD
}

\author{
From Birds, Moulds and Atoms: Toward a \\ Post-human Notion of Expressivity
}

\author{
Simón Díez-Montoya \\ diez.simon@urosario.edu.co. \\ Universidad del Rosario (Colombia)
}

Fecha de recepción: 11/11/ 2014

Fecha de evaluación: 24/02/2015

Fecha de aprobación: 15/09/2015

\section{Resumen}

El artículo propone algunas formas de ampliar el rango del concepto de expresividad, para mostrar cómo este ejercicio abre un campo fronterizo entre el arte, la filosofía y la ciencia. Así, primero revisa una tesis de Gilles Deleuze y Félix Guattari, según la cual hay arte en los animales, y la presenta como una defensa de la expresión autónoma en ellos, para luego examinar el problema más general de la consistencia de la expresión; en segundo término, amplía esta tesis argumentando que no solo los animales, sino también los organismos unicelulares, pueden contribuir a la expansión del concepto de expresividad, caso que se demuestra a través de un ejemplo emparentado con el plano de expresión: ver cómo la conducta del moho mucilaginoso puede interpretarse como creativa; por último, se arguye que la ontología deleuziana, tal como es reconstruida por Manuel DeLanda, al dar una nueva imagen del mundo, puede sentar algunas bases para una concepción materialista de creación que pudiese deterritorializar la expresividad más allá de la esfera humana.

Palabras clave: Deleuze, DeLanda, Expresividad, Ereatividad, Materialidad, Ontología. 


\begin{abstract}
This paper proposes some ways of expanding the expressivity concept range, in an attempt to show how this exercise opens a frontier-space between art, science, and philosophy. With this in mind, first, it revises a thesis, taken from Gilles Deleuze and Félix Guattari, according to which there is art in animals, and then presents it, as a defense of their autonomous expression; this, in order to examine the more general problem of the consistency of expression. Second, it expands this thesis by arguing that not only animals, but also unicellular organisms can contribute to the expansion of expressivity; this is showed through an example akin to the plane of expression: by seeing how the conduct of the slime mould can be interpreted as creative. Lastly, it argues that adeleuzian ontology, as presented by Manuel DeLanda, by bringing forth a new image of the world, which can supply a basis for a materialist conception of creation that could potentially deterritorialize expressivity beyond the human sphere.
\end{abstract}

Key words: Deleuze, DeLanda, Expressivity, Creativity, Materiality, Ontology.

Se ha creído que el territorio de un plano de expresión complejo pertenece solo a la especie humana, y la creatividad ha sido paradigmáticamente asociada a lo que puede lograr una persona con sistema nervioso central; frente a esa creencia, la tarea del presente artículo ha de verse como la de desterritorializar el plano de expresión del ámbito de la humanidad y reterritorializarlo drásticamente ampliado. La idea es concebir la expresividad como un concepto absolutamente transversal a la materialidad del mundo, que tiene instanciaciones desde las partículas más primordiales hasta las fuerzas cósmicas que moldean el universo; esta visión de mundo esboza posibilidades de trabajo en zonas limítrofes entre el arte, la ciencia y la filosofía, y, de igual forma, constituye una invitación a buscar caminos de pensamiento más allá de la esfera humana. Por todo esto, es probable que el problema de fondo en realidad no sea solo la expresividad, sino una visión de mundo limitada que hasta ahora ha monopolizado el uso de ciertos conceptos.

El texto se divide en tres partes principales, que abarcan, respectivamente, tres niveles de análisis: lo animal (macro), lo orgánico unicelular (meso) y lo atómico (micro). Comienzo examinando una tesis de Deleuze y Guattari que, al mostrar la aplicación de una teoría de la expresividad extendida, abre todo el problema de pensar más allá de lo humano; allí lo crucial es ver cómo en la constitución de un territorio está en juego el arte mismo. Luego, me concentro en el nivel de los organismos unicelulares, para 
mirar el remarcable caso del moho mucilaginoso, un ameboide, según arguyo, creativo; lo particular del caso es que muestra muy bien cómo una expansión conceptual puede ser totalmente productiva, en primera instancia, para las ciencias, pero también para la filosofía. Finalmente, termino con la exposición que hace DeLanda del concepto de multiplicidad en la ontología deleuziana, donde se exhibe también el trasfondo técnico detrás de este; lo anterior permite observar cómo los compromisos ontológicos moldean la esfera de lo concebible, en tanto, bajo esta ontología, la materia como tal puede ser vista como autónoma y creadora de formas.

Según Deleuze y Guattari, el arte es, sobretodo, un producto de la vida misma y no un ejercicio humano; con esto en mente, analizo esta tesis a partir de tres ejes: (i) la relación entre la génesis del territorio y la génesis del arte; (ii) el movimiento territorialartístico que va de la marca y la firma al estilo, y (iii) el planteamiento del problema de la consistencia. Para Deleuze y Guattari, el territorio surge del caos ${ }^{1}$, i.e, el medio de todos los medios donde nacen los medios y los ritmos (2010, p. 319). Mientras los medios son bloques de espacio-tiempo constituidos por la repetición periódica (código) de sus componentes, los ritmos son las coordinaciones y las comunicaciones (transcodificaciones) entre estos bloques (Deleuze \& Guattari, 2010, p. 320). Los medios y ritmos pueden hallarse en todo organismo, siempre estructurado a partir de cuatro medios principales y sus interacciones: un medio-exterior de materiales, un medio-intermedio de membranas y límites, un medio-interior de elementos constitutivos y un medio-anexado de fuentes de energías y acciones-percepciones. El ritmo, tal como lo ven los autores, es un 'entre-dos': el paso de la noche al día, de lo inorgánico a lo orgánico, de la planta al animal, del animal al animal humano: básicamente, hay ritmo desde el momento en que hay paso transcodificado de un medio a otro, comunicación de medios, coordinación de espacios-tiempos heterogéneos" (Deleuze \& Guattari, 2010, p. 320). En últimas, es el paso de un medio a otro; por eso, tal como la repetición periódica produce la diferencia, así también la interacción periódica de medios produce ritmo. Más aún, siempre que hay ritmo y transcodificación, esto es, coordinación o comunicación entre bloques

1 A propósito, Elizabeth Grosz señala que el caos son: "los movimientos vertiginosos e impredecibles de fuerzas y vibraciones oscilantes que constituyen el universo. El caos entendido no como el desorden absoluto, sino más bien como una plétora de órdenes, formas y voluntades -fuerzas que no pueden ser distinguidas o diferenciadas unas de otras $[\ldots]$ para ser otra cosa, donde tanto lo actual como lo virtual son indistinguibles" (Grosz, 2008, p. 5). 
de espacio-tiempo, hay más que una simple cópula: se trata de la constitución de un nuevo plano, un superávit material (Deleuze y Guattari, 2010, p. 321). Deleuze y Guattari se apoyan en esta teoría de transcodificaciones, de von Uexkiill, para dar cuenta de lo que podría llamarse la naturaleza 'excitable' del mundo; el hecho de que, por ejemplo, las plantas y los animales tengan células excitables que responden a diferencias intensivas, como la temperatura y la presión.

De esta forma, el territorio nace como producto de la territorialización de los medios y ritmos. Igual que en el organismo, en el territorio "hay una zona interior de domicilio o de abrigo, una zona exterior de dominio, límites o membranas más o menos retractiles, zonas intermediaras o incluso neutralizadas, reservas o anexos energéticos" (Deleuze y Guattari, 2010, p. 321). Pero lo clave es que el territorio surge cuando los componentes del medio se vuelven más que direccionales para tornarse dimensionales, y cuando se vuelven más que funcionales para tornarse expresivos". La emergencia de materias de expresión (cualidades) es la que va a definir el territorio" (Deleuze y Guattari, 2010, p. 321). Es importante notar que la teoría de la expresión propuesta por los autores liga fundamentalmente la expresividad con la territorialización: crear un territorio es la expresión más básica, a la vez que los componentes expresivos son territorializantes. Un claro ejemplo de esto, como notan Deleuze y Guattari, proviene de los animales territoriales; en su caso, pueden convertir la orina y las heces, que hasta ese momento no eran más que desechos materiales de la cadena alimenticia, en marcas ofirmas que expresan un dominio. "Sonido, color y demostraciones conductuales que involucran posturas, movimientos, ritmos y siluetas, son ejemplos de marcadores territoriales expresivos, a veces utilizados en competencias entre machos, a veces en rituales de cortejo con las hembras" (DeLanda, 2005, p. 122). El proceso de territorialización se da cuando el medio se vuelve cualidad, y el ritmo se torna expresivo. No es el territorio el que precede a la marca, sino viceversa: marcar es territorializar. Más aún, Deleuze y Guattari se preguntan si esta emergencia de materias de expresión no es el arte mismo. Apropósito comenta Deleuze:

Construir un territorio es casi, para mí, el nacimiento del arte. Lo que interviene en el marcaje de un territorio son también una serie de posturas, por ejemplo, agacharse, levantarse; una serie de colores [...] Los mandriles, el color del trasero de los mandriles, pone de manifiesto la frontera del territorio. Color, campo, postura. Son las tres determinaciones del arte. El color, las líneas, ¡las posturas de animales son a veces verdaderas líneas! Color, línea, campo. Es el arte en estado puro(1996). 
Así que el primer movimiento es marcar o firmar el territorio para establecer un dominio, una morada. El artista, dicen Deleuze y Guattari, es el primero en fijar una frontera, por lo cual el ave de emparrado (Ptilonorhynchidae) es un verdadero artista en este sentido; estos pájaros pueden construir nidos sumamente complejos para atraer a las hembras, en los cuales los componentes cromáticos de la construcción son inversamente proporcionales a las características de su plumaje; se podría hablar de una conducta constructiva asociada a un principio de complementariedad del azul con el marrón. En especial, hay una especie que tiene un plumaje azul satinado que cubre todo su cuerpo; esta es la que menos trabajo hace, construye nidos medianos con algunas decoraciones que consisten en uno que otro objeto azul por ahí. Hay otra especie que tiene un plumaje marrón, a excepción de sus alas, que son azules, la cual construye nidos más grandes y los decora con cualquier objeto azul que encuentre por ahí. Pero hay otra especie que no tiene ni un rastro de azul en su plumaje marrón; sin embargo, construye unos nidos impresionantes, tanto por sus complejidades arquitectónicas (torres, techos, arcos), como por la forma como los decora: llenos de comida, flores, hongos, en realidad, cualquier cosa que encuentren por ahí. Aquí se evidencia el 'superávit de expresión’ que mencionaba más arriba, ya que en la construcción de un nido de un ave de emparrado se conjugan muchos factores más que los meramente funcionales, hay un juego de cortejo intricado, que requiere de demostraciones de habilidad, que a su vez exigen capacidades arquitectónicas grandes. A partir de este ejemplo se puede pensar "que hay un automovimiento de las cualidades expresivas (y que) la expresividad no se reduce a los efectos inmediatos de un impulso que desencadena una acción en un medio [...]. Por el contrario, las cualidades expresivas [...] son auto-objetivas, es decir, encuentran una objetividad en el territorio que trazan (2010, p. 323). En definitiva, que el arte no es privilegio del hombre.

¿Qué puede significar que las cualidades expresivas sean auto-objetivas? Una interpretación plausible es ver en la auto-objetividad una especie de autosostenimiento de la materia de expresión (similar a lo que Deleuze y Guattari llamaran consistencia o consolidación). Esto tendría sentido en la medida que se liga con otra tesis de los autores, según la cual "el artista crea bloques de perceptos y afectos, si bien la ley de la creación es que el compuesto debe sostenerse por símismo" (Deleuze y Guattari, 1994, p. 164). Algo así como que "el arte, propiamente, emerge cuando la sensación puede desligarse y cobrar autonomía de su creador y observador, cuando algo del caos desde el cual surge puede respirar y tener vida por sí mismo” (Grosz, 2008, p. 7). 
Con esto estamos al límite del segundo movimiento del arte, el paso de la marca o firma (el cartel o pancarta) al estilo. El estilo surge, por un lado, cuando ciertas cualidades expresivas comienzan a relacionarse entre sí para constituir motivos territoriales, lo cual está fundamentalmente ligado a la expresión de impulsos internos; y, por otro lado, cuando ciertas cualidades expresivas comienzan a relacionarse entre sí para constituir contrapuntos territoriales, lo cual está íntimamente ligado al posicionamiento respecto a circunstancias exteriores (Deleuze y Guattari, 2010, p. 324). De esta forma, el estilo se define por la posibilidad de explotar las potencialidades de interacción con un medio interior (impulsos) o con un medio exterior (circunstancias); esto hace que las cualidades expresivas se vuelvan más que meras marcas; aquí también surgen personajes y paisajes.

Asimismo, Deleuze y Guattari se enfocan en mostrar el territorio como el primer agenciamiento, esto es, como una expresión de la organización interna entre individuos heterogéneos puestos en conjunto, pero no homogeneizados. El territorio es tanto infra-agenciamiento (cartel o pancarta) como intra-agenciamiento (motivos y contrapuntos); pero estos agenciamientos también pueden verse como pasajes entre otros agenciamientos; un buen ejemplo es observar el intra-agenciamiento del pájaro reyezuelo (Troglodytidae):

[El]macho toma posesión de su territorio y produce un "ritornelo de caja de música", como defensa contra los posibles intrusos; construye nidos en ese territorio, a veces hasta una docena; cuando llega la hembra, se pone delante de un nido, la invita a visitarlo, deja sus alas suspendidas, baja la intensidad de su canto, que en ese momento queda reducido a un solo trino. Se pone así de manifiesto que la función de nidificación está fuertemente territorializada, puesto que los nidos son preparados exclusivamente por el macho antes de la llegada de la hembra, que sólo los visita y los acaba; la función de "corte" está igualmente territorializada, pero en menor grado, puesto que el ritornelo territorial cambia de intensidad para hacerse seductor (Deleuze y Guattari, 2010, p. 329).

En este caso, el intra-agenciamiento territorial de motivos y contrapuntos comienza a desterritorializarse a favor de un agenciamiento-cortejo de seducciones y posturas. De este modo, comienza a vislumbrarse el problema de la consistencia: ¿cómo se mantienen en pie, por sí mismos, los elementos heterogéneos de un agenciamiento? y, más aún, ¿cómo se mantienen en pie, por sí mismos, varios agenciamientos heterogéneos? Deleuze y Guattari afirman que "la respuesta más clara, más fácil, la proporciona un modelo arborescente, centralizado, jerarquizado, lineal, formalizante" 
(2010, p. 333). Se refieren al modelo de Timbergen, quien concibe un esquema centralizado y modular de los agenciamientos: primero se activa el sistema central, luego los impulsos se transmiten a un sistema más bajo, y así sucesivamente. Sin embargo, los autores abandonan este modelo a favor de un modelo rizomático. La estructura formal, central, jerarquizada y trascendente de lo arborescente contrasta con la estructura material, descentralizada, desjerarquizada e inmanente de lo rizomático. En efecto, "no se hablará tanto de automatismo de un centro superior como de coordinación entre centros y agrupamientos celulares o poblaciones moleculares que efectúan esos acoplamientos: no hay una forma o una buena estructura impuesta, ni desde afuera ni desde arriba, sino más bien una articulación por dentro, como si moléculas oscilantes, osciladores, pasaran de un centro heterogéneo a otro" (Deleuze y Guattari, 2010, p. 333).

La teoría de la consolidación de Dupreel, que citan Deleuze y Guattari, no describe más que la productividad de la diferencia o, en términos de Dupreel, el paso de la materia difusa a los agregados delimitados; en este sentido, la consolidación es esencialmente un proceso creativo. "El agenciamiento territorial es un consolidado de medio, un consolidado de espacio-tiempo, de coexistencia y sucesión" (Deleuze \& Guattari, 2010, p. 334); es la elaboración de un material rico a partir de diferencias intensivas de fuerzas.

Vale la pena detenerse exclusivamente sobre el tercer aspecto diferenciador de la consistencia, teniendo en cuenta las relaciones moleculares-molares y no tanto los temas melódicos, ni el poder de lo natal. Deleuze y Guattari sugieren que hablar de materias de expresión hace evidente que lo expresivo está en una relación con la materia, y las materias de expresión consistentes constituyen, a su vez, sistemas semióticos y agregados materiales. DeLanda, por ejemplo, considera que es posible equiparar los patrones expresivos con “información” utilizada en un sentido técnico como patrones físicos asignificantes y no-lingüísticos, e.g., los fotones: "La información física invade al mundo y es a través de su continua producción que puede decirse que la materia se expresa a sí misma" (2005, p. 122). En efecto, DeLanda está alineado con Deleuze y Guattari cuando propone como ejemplos de la expresividad física las "huellas" de átomos (trazos de radiación que pueden ser modelados por un espectro de colores) y los patrones químicos de los organismos (el ADN, como eje central de la evolución, demuestra un potencial expresivo enorme: millones de patrones de aminoácidos capaces de crear formas de vida completamente diversas). Como aseveran Deleuze y Guattari: "la consistencia, lejos de estar reservada a formas vitales complejas, concierne ya plenamente al átomo y a las partículas más 
elementales" (2010, p. 340). Tal vez es en este orden que Deleuze y Guattari afirman que la vida misma presenta un superávit de diferenciaciones productivas. Todo el proceso que hemos recorrido lo recoge muy bien Grosz cuando afirma que:

El arte viene del exceso, en el mundo, en los objetos, en las cosas vivientes, que les permiten ser más de lo que son, de dar más que sí mismos, sus propiedades materiales y cualidades, sus posibles usos, más de lo que es autoevidente. El arte es la consecuencia de ese exceso, esa energía o fuerza, que pone la vida en riesgo por mor de la intensificación, por mor de la sensación en sí misma [...] para lo que puede ser magnificado, intensificado; para lo que es más, a través de lo cual la creación, el riesgo y la innovación se sobrellevan por sí mismos; para cómo y qué pueden intensificar(2008, p. 63).

De todo lo anterior queda al menos una conclusión pertinente para la creación: es posible tomar cualquier cosa y convertirla en materia de expresión. También quedan abiertas varias potencialidades si la expresión deja de anclarse en lo humano nada más y se extiende hasta nuevos ámbitos. Como bien nota DeLanda: "la materia se expresa en muchas formas, desde la simple emisión de información física hasta el uso deliberado de melodía y ritmo. El universo mismo puede ser visto como una gran sinfonía de materialidad expresiva" (2005, p. 123). En este sentido, sería relevante una desterritorialización conceptual para ampliar la esfera de la expresividad.

\section{II}

Abrir caminos a través de la maleza del antropocentrismo no es fácil. La creatividad sigue siendo un concepto que supuestamente requiere como condición de su aplicación un contexto cultural poblado de agentes racionales; esto implica un reto y una posibilidad para múltiples centros de invención: mostrar caminos que lleven a revaluar cómo aplicamos este concepto. En esta sección quisiera presentar un ejemplo con el cual enfrento el reto; arguyo, con ayuda de otros autores, que el comportamiento de un organismo tan simple como el moho mucilaginoso puede interpretarse como creativo.

El moho mucilaginoso, o Physarumpolychephalum, es un ameboide unicelular multinucleado y amarillento constituido por una red de tubos protoplásmicos oscilantes que puede resolver laberintos (Nakagaki, 2001), construir redes de navegación y servir como modelo de computación paralela (Nakagaki, Kobayashi, \& Tero, 2004), (Nakagaki, Yamada, \& Hara, 2001), imitar diseños de autopistas 
(Adamatzky y Alonso-Sanz, 2011) y ser considerado como expresión de creatividad (Adamatzky, Armstrong, Jones, y Gunji, 2013). De entrada, es evidente el contraste entre la simplicidad material del moho y su comportamiento complejo. Por eso mismo, este rudimentario organismo desafía el prejuicio filosófico-científico según el cual hay una especie de 'escalera de la evolución'; donde los animales con sistema nervioso y límbico son considerados el escalafón 'superior', y todo lo que no cumpla estas condiciones es considerado 'inferior'. La racionalidad, la inteligencia, la lógica, el pensamiento y la creatividad, por ejemplo, se toman comúnmente como características exclusivas del primer grupo. La idea es comenzar a diluir este prejuicio estudiando el moho mucilaginoso.

Hay una investigación ${ }^{2}$ sobre computación no-convencional que quisiera explorar a fondo, puesto que defiende que la conducta del Physarum puede interpretarse como creativa. La objeción inmediata que podría aparecer es que la creatividad tiene, al menos, dos condiciones de emergencia necesarias, un trasfondo cultural y un sujeto formado: sin cultura y sin mentes, ¿cómo se puede hablar de creatividad? Sin embargo, los autores reclaman que la creatividad cuenta con varias características externas analizables, a partir de cuatro criterios formales para determinar la creatividad (hay más, aunque no serán revisados): (i) pensamiento divergente, (ii) asociaciones remotas, (iii) flexibilidad y (iv) adaptabilidad novedosa. Según los autores, es posible identificar estos aspectos en el moho (Adamatzky, Armstrong, Jones, \& Gunji, 2013).

Dada su heterogeneidad, lo que puede hacer el 'cuerpo' Physarum es sorprendente; tiene un 'pensamiento' divergente, en tanto puede reaccionar a interacciones de un medio de atrayentes y repelentes (i.e., puede buscar comida y evitar amenazas eficientemente); tiene la capacidad de variar sus oscilaciones internas para cambiar el flujo de materia que pasa por los tubos protoplásmicos, covariando con presiones del ambiente (Nakagaki, Yamada, y Tóth, 2001, p. 49); puede formular asociaciones remotas, en la medida que responde físicamente a la información espacial de su entorno (esto difiere de lo anterior, dado que aquí se está destacando la capacidad del moho de reconocer un espacio y desplegarse por él) (Adamatzky, Armstrong, Jones, \& Gunji, 2013, p. 447); cuenta conflexibilidad, si esta se entiende como la posibilidad de generar crecimiento espontáneo: el moho puede llevar tubos protoplásmicos a regiones no exploradas, incluso si ya cuenta con fuentes de nutrientes (algo así como continuar resolviendo una tarea que ya ha sido resuelta) (Adamatzky,

2 Me refiero a la de Adamatzky, A., Armstrong, R., Jones, J., \& Gunji, Y. (2013). On creativity of slime mould. International Journal of General Systems, 42 (5), 441-457. 
Armstrong, Jones, y Gunji, 2013, p. 449); demuestra adaptabilidad novedosa a la hora de enfrentar problemas para conseguir alimentos, como recorrer un laberinto. Para Nakagaki, por ejemplo, el moho evidencia una especie de 'inteligencia' primitiva en la 'resolución' de laberintos, porque es capaz de desplegar mecanismos de supervivencia en situaciones complejas (2001, p. 769). El método que utiliza el moho para conectar dos fuentes de comida (una a la entrada y la otra a la salida del laberinto) es siempre similar y consiste en expandir sus tubos protoplásmicos a lo largo de todo el laberinto, y luego seleccionar el tubo que presente el flujo de materia más eficiente (que siempre resulta siendo en camino más corto para resolver el laberinto). Según otros, el moho también presenta una suerte de 'memoria espacial externa', en la medida que nunca recorre caminos que ya ha explorado (Reid, Latty, Dussutour, \& Beekman, 2006, p. 460). Al expandir sus tubos protoplásmicos, el moho deja una mucosa extracelular que parece 'marcar' las áreas ya recorridas, debido a que cada vez que se encuentra con esta mucosa busca otro camino.

Un camino de exploración que surge a partir de esto es el de ver al moho poseyendo algo así como un 'pensamiento' táctil. Hay investigaciones científicas que consideran que el moho ofrece un modelo plausible para pensar problemas como la emergencia del pensamiento, la memoria y la creatividad, ya que muestra cómo un organismo unicelular no-neural exhibe comportamientos complejos asociados con conductas típicamente atribuidas a organismos neurales (Adamatzky, Armstrong, Jones, \& Gunji, 2013). En otra área experimental, el Physarum está siendo utilizado para estudiar formas de computación no-convencionales (e.g., la computación paralela), dado que no tiene un sistema de procesamiento central (cerebro) (Tsuda, Aono, y Gunji, 2004); incluso, ha sido utilizado para comprobar la eficiencia de redes de transporte, por su capacidad de formar siempre el camino más corto para hacer el flujo de materia más eficiente (Adamatzky y Alonso-Sanz, 2011).

Todo esto también puede servir como puerta de entrada a una nueva concepción sobre la materialidad, en tanto muestra cómo organizaciones aparentemente simples de materia pueden propiciar desarrollos y comportamientos emergentes complejos. La idea sería ampliar la lupa y entrar a considerar flujos de materia y energía más diminutos, ¿qué procesos similares hay a nivel molecular o celular? Lo cual puede ayudar a disipar otro prejuicio filosófico-científico: considerar la materia como inerte y a la espera de formas externamente impuestas; pues obliga a repensar un mundo donde la materia exhibe una complejidad y vivacidad inmanente (DeLanda, 2002, p. 5). 
En esta sección quiero detallar un aspecto en particular de la reconstrucción que hace DeLanda de la ontología deleuziana; bajo su interpretación, se puede considerar a Deleuze un materialista y un realista en sentido fuerte, ya que le otorga completa autonomía al mundo como tal (no hace de las mentes una condición necesaria para su existencia plena). Una cuestión central para una ontología realista es que debe dar cuenta del problema general de la emergencia de la forma en las entidades (la morfogénesis); lo cual, a su vez, requiere una explicación de los procesos de individuación y conservación de la materia sin apelar a arquetipos ideales externos, en vista de que no basta con negar la trascendencia y afirmar la inmanencia: algo tiene que reemplazar las esencias (DeLanda, 2002, p. 2). Mientras una ontología de esencias trascendentes funciona con un principio de identidad, a saber, que la materia tiene forma porque es la copia, que varía de acuerdo a grados de perfección, de un modelo o esencia externo e inmutable, una ontología de procesos dinámicos inmanentes funciona con un principio de diferencia, esto es, que la materia tiene forma porque es el resultado procesos de diferenciación intensivos productos de diferencias críticas en, por ejemplo, la temperatura, la presión o la velocidad que afectan la formatividad.

No obstante, ¿con qué propósito hacer todo esto? Como bien señala DeLanda, una comprensión más profunda del mundo de Deleuze puede iluminar otras áreas de su trabajo. A propósito comenta: "el trabajo de Deleuze, desde el comienzo, se ha preocupado tanto por la matemática y la física, como por el arte. Pero me parece que solo cuando entendemos el mundo deleuziano de flujos materiales y energéticos, y las formas que emergen espontáneamente de estos flujos, podemos comenzar a preguntar "¿qué es una novela o una pintura o un pedazo de música?" en este mundo" (DeLanda, 2002, pp. 5-6). En este caso, habría que preguntarnos qué es la expresividad y cómo se entiende la creatividad en este mundo. A continuación presento, con la ayuda de DeLanda, el eje central de la ontología deleuziana: el concepto de multiplicidad.

En la ontología de Deleuze, el concepto de multiplicidad reemplaza al de esencia. La esencia de una cosa se ha pensado tradicionalmente como aquello sin lo cual esa cosa no sería lo que es; así, los objetos que se parecen unos a otros lo hacen porque tienen la misma esencia, formando una clase natural. Por el contrario, bajo una ontología deleuziana, una cosa no se define por su esencia, que es ideal y externa, sino por el proceso diferenciador que la produjo, que es material e interno. 
En vez de apelar a arquetipos que trascienden el mundo de la materia y la energía, una ontología deleuziana deberá explicar la morfogénesis a partir de procesos inmanentes a la materia y la energía (DeLanda, 2002, p. 10). No obstante, para ver la importancia del concepto de multiplicidad hay que estudiar su herencia conceptual, que viene, sobretodo, de la matemática.

El concepto de multiplicidad está relacionado con un término que viene de la geometría diferencial: variedad [manifold]. La geometría diferencial, desarrollada por Gauss y Riemann, ofrece una forma de adaptar los procesos utilizados en el cálculo integral al estudio de la superficie como un espacio en sí mismo (DeLanda, 2002, pp. 1112). Esto hace posible el estudio de una superficie bidimensional sin la necesidad de desplegarla sobre un plano coordenado tridimensional, lo cual se logra concibiendo una superficie de puntos infinitesimales donde las relaciones entre los puntos se determinan a partir de ecuaciones diferenciales. Así, indica DeLanda, "una multiplicidad deleuziana toma como elemento definitorio estos dos aspectos de una variedad: su número variable de dimensiones y, más importante, la ausencia de una dimensión suplementaria (más alta) que impone una coordinación extrínseca, y por tanto, una unidad extrínsecamente definida" (2002, pp. 12-13). Lo importante allí es la posibilidad de concebir una organización carente de unidad trascendental. Mientras las esencias requieren tanto un plano ideal (más alto), donde están contenidas, como una unidad definitiva (e.g., los humanos como animales donde se da la unidad de animalidad y racionalidad), las multiplicidades, como las variedades, no necesitan un plano superior ni una unidad definitiva para formar un sistema organizado (DeLanda, 2002, p. 13).

Lo clave es que la variedad sirve para modelar sistemas físicos. Comenzando con la teoría de sistemas dinámicos, DeLanda explica cómo es posible estudiar los cambios en el comportamiento de un objeto físico trazando su trayectoria en una variedad, i.e., el espacio de posibles estados del sistema (2002, p. 13). Cada estado del objeto, o estado espacial [statespace], es representado por un punto en la variedad. Los posibles cambios de estado del objeto, o grados de libertad, corresponden a las dimensiones de la variedad. Los cambios de un estado a otro describen una trayectoria particular, que en vez de describir propiedades dan cuenta de un proceso físico. Para explicar patrones de comportamiento comunes a muchos procesos físicos, se pueden utilizar recursos topológicos (como los diseñados por Poincaré) para mostrar que las trayectorias tienden a dirigirse hacia singularidades que funcionan como atractores [attractors] de materia en el sistema, los cuales representan estados finales para las trayectorias. En vista de que varias trayectorias 
pueden confluir en el mismo estado final, las singularidades vendrían a representar las tendencias a largo plazo e inmanentes del sistema, o los estados que el sistema tenderá a adoptar espontáneamente en tanto no sea constreñido por otras fuerzas (DeLanda, 2002, p. 15). Por ejemplo, propone DeLanda, la formación de burbujas de jabón y de cristales de sal, a pesar de que son fenómenos completamente distintos, puede verse como el producto de componentes internos buscando un punto de energía libre mínima:

la forma esférica de una burbuja de jabón [...] emerge de las interacciones entre sus moléculas constituyentes mientras éstas son constreñidas energéticamente para "buscar" el punto donde la tensión de superficie se minimiza [...] [Asimismo], el mismo punto mínimo puede guiar los procesos que generan otras formas geométricas. [Si] en vez de moléculas de jabón tenemos los componentes atómicos de un cristal de sal ordinario, la forma que emerge de la energía minimizadora [...] es un cubo (DeLanda, 1998, p. 1).

El punto es que la misma forma topológica, que es un estado final inmanente al espacio de posibilidades de los agregados moleculares, guía dos procesos de morfogénesis distintos, sin apelar a la esencia de la 'burbujeidad' o 'cristalidad'. Esto es central en vista de que

las singularidades, al determinar tendencias a largo plazo, estructuran las posibilidades que conforman el estado espacial, y, por extensión, estructuran las posibilidades abiertas al proceso físico modelado por el estado espacial. Además, las singularidades [...] tienden a caracterizar procesos independientemente de sus mecanismos físicos particulares [...]. Esta independencia-de-mecanismo es lo que hace que las singularidades [...] sean perfectas candidatas a reemplazar las esencias" (DeLanda, 2002, p. 16).

Para DeLanda, esto puede verse como una forma de pensar topológicamente las cosas; lo que posibilita, a través de instrumentos representacionales, la visualización del devenir a manera de trayectoria.

Dado que, a diferencia de las esencias, que adecúan arquetipos preestablecidos a la materia, las multiplicidades se definen por conjuntos de singularidades que se desarrollan progresivamente, a través de procesos diferenciadores que le dan forma a la materia, es necesario explicar cómo pueden darse dichos procesos en sistemas físicos. DeLanda muestra que usando recursos de la teoría de grupos se puede dar 
cuenta de la diferenciación progresiva para transformaciones geométricas (2002, p. 17). Esto sirve igualmente para dar cuenta de la diferenciación progresiva en sistemas físicos a partir de transiciones de fase. La teoría de grupos ofrece la posibilidad de relacionar conjuntos de entidades a partir de reglas de combinación que generan entidades que hacen parte del mismo conjunto. Para explicar las diferenciaciones progresivas se requiere que las entidades de estos conjuntos no sean objetos, sino transformaciones que se evalúan de acuerdo con criterios de invariabilidad y simetría. Por ejemplo, dado que un observador no podría diferenciar el estado final de un cubo que sufre transformaciones de $90^{\circ}$, se diría que el cubo permanece invariante; en contraste, mientras que una esfera permanece invariante ante transformaciones de $45^{\circ}$, un cubo no, por lo cual se diría que la esfera tiene más simetría relativa al cubo (permanece invariante a más transformaciones). Como aquí se están relacionando los conjuntos de transformaciones de dos entidades, la teoría de grupos permite concebir procesos en los que una entidad se convierte en la otra a partir de procesos de pérdida o ganancia de simetría (DeLanda, 2002, p. 18). Así, una esfera podría 'devenir cubo' al dejar de ser invariante frente algunas transformaciones; a esto se le llama una transición rompedora de simetría (DeLanda, 2002, p. 18). En los sistemas físicos, las transiciones de fase, i.e., las diferencias críticas que cambian un sistema físico de un estado a otro, pueden ser vistas como procesos simétrico-rompedores: e.g., puesto que un gas permanece invariante a más transformaciones que un sólido, y por tanto es más simétrico, se puede pensar en un proceso simétrico-rompedor que lleve el gas a un estado sólido a partir de una cascada de sucesivos quebramientos de simetría.

Mas, ¿cuál es la relación de esto con las multiplicidades? DeLanda muestra que hay procesos simétrico-rompedores en la conversión de una singularidad a otra. Las transiciones de fase pasan a ser ahora bifurcaciones, determinadas por diferencias intensas en algunos estados espaciales, medidas en valores críticos. Tal como los atractores representaban las tendencias a largo plazo de las trayectorias, así también las bifurcaciones pueden establecer las secuencias recurrentes de las formas topológicas (DeLanda, 2002, p. 20); este procedimiento abstracto puede replicarse en sistemas físicos que sobrelleven transiciones de fase a partir de procesos simétricorompedores. Por ejemplo, pueden estudiarse diversos flujos hidrodinámicos analizando un fenómeno simple como hervir agua, que pasa por tres estados característicos: conducción-convección-turbulencia (DeLanda, 2002, p. 20). Al hervir un contenedor de agua, esta comienza calentándose de abajo hacia arriba de manera simple y constante (conducción); luego de cierto cambio crítico en la 
temperatura, el flujo estable desaparece y es reemplazado por la formación de pequeños torbellinos (convección); pasado otro punto crítico en la temperatura, desaparece el flujo periódico, y el flujo asume un estado caótico (turbulencia). Todo este proceso puede verse como la sucesión de una cascada de bifurcaciones simétrico-rompedoras, en el cual cada flujo, al perder simetría, comienza a cambiar. Lo importante es que cada uno de esos flujos, aunque las relaciones de sus componentes internos sean distintas, repite la misma secuencia de: estado espacialvalor crítico-bifurcación. Así: "A diferencia de la generalidad de las esencias [...] la universalidad de una multiplicidad es típicamente divergente: las diferentes realizaciones de una multiplicidad no guardan semejanza alguna [con el producto final] y no hay, en principio, un final al conjunto de formas divergentes potenciales que puede adoptar [...].[Las] multiplicidades le dan forma a procesos, no al producto final, así que los resultados finales de procesos que realizan la misma multiplicidad pueden ser altamente disímiles entre sî' (DeLanda, 2002, p. 22). Este arsenal teórico permite pensar la diferencia como productora de eventos de morfogénesis.

DeLanda explica que Deleuze ve estas multiplicidades como coexistiendo unas con otras a manera de un manojo poco diferenciado en un continuo virtual o plano de consistencia; pero este estadio virtual no es una especie de cielo trascendente de esencias ideales, sino algo que hace parte del mundo físico de átomos y moléculas; esto se hace más asible cuando se especifica cuál es la relación entre lo virtual y lo actual. Para Deleuze, dice DeLanda, cuando hablamos de lo "real" nos referimos, normalmente, al mundo como nosotros lo experimentamos: un mundo con un espacio extensivo (i.e. divisible y cuantificable en centímetros, metros y kilómetros) y con un tiempo extensivo (i.e. divisible y cuantificable en segundos, minutos y horas). Este sería el estadio de lo actual, extensivo espacio-temporalmente; en términos de Deleuze, una ilusión objetiva. Sin embargo, para Deleuze estas formas extensivas son solamente flujos coagulados y desacelerados, son el producto final de procesos intensivos inmanentes a flujos materiales y energéticos, que le dan forma a la materia. En este sentido, lo "real" no solo comprende el mundo de lo actual experimentado, sino también el mundo virtual de multiplicidades que animan las diferenciaciones progresivas que están presentes en las cascadas simétrico-rompedoras que generan burbujas de jabón y cristales de sal. Una forma simplificada de decirlo sería que lo actual captura el producto final, mientras lo virtual captura los procesos que hacen posible ese producto final. Deleuze, aunque abre otras preguntas, asevera, en Diferencia y Repetición, que: 
Lo virtual $[\ldots]$ no se opone a lo real; posee una plena realidad por sí mismo. [...] [La] actualización de lo virtual siempre se hace por diferencia, divergencia o diferenciación. La actualización rompe tanto con la semejanza como proceso, como con la identidad como principio. Nunca los términos actuales se asemejan a la virtualidad que actualizan; las cualidades y las especies no se parecen a las relaciones diferenciales que encarnan; tampoco las partes se asemejan a las singularidades que encarnan (Deleuze, 2002, pp. 118-119).

Las implicaciones que esto tiene no solo se extienden a una consideración distinta de las entidades físicas, sino que llevan a una nueva forma de pensar lo que hay en el mundo. Deleuze quiere evitar tanto un pensamiento esencialista (arquetipos ideales preformados) como uno tipológico (categorizaciones bajo criterios formales), porque cree que ambos presuponen un universo poblado de individuos completamente formados. "La alternativa que ofrece Deleuze es evitar tomar como dados individuos completamente formados, o lo que es lo mismo, siempre dar cuenta de la génesis de los individuos vía procesos de individuación específicos, como el proceso de desarrollo que transforma un embrión en un organismo", recalca DeLanda (2002, p. 40). En suma, si una ontología de esencias concibe la materia como un receptáculo pasivo que recibe su forma con base en arquetipos ideales, heredados de un estadio trascendental, una ontología de procesos dinámicos, en cambio, le adjudica a la materia misma una autonomía para generar formas que no son copias de un ideal trascendental, sino el resultado de diferenciaciones progresivas inmanentes a flujos materiales y energéticos.

El último elemento del trasfondo técnico del concepto de multiplicidad ayuda a dar cuenta de esta relación entre lo virtual y lo actual. En términos abstractos, lo virtual y lo actual pueden ser modelados en una variedad que incluya un retrato de fases y un campo de vectores, y que esté poblada de trayectorias (DeLanda, 2002, p. 31). Mientras el retrato de fases captura todas las trayectorias actualizadas, el campo de vectores captura todas las potencialidades de las trayectorias que podrían ocurrir. Así, los pocos puntos singulares incluidos en las trayectorias actualizadas contarían notablemente con una cantidad casi infinita de puntos que comprenden las trayectorias virtuales. Como explica DeLanda:

Mientras una trayectoria particular (o curva integral) modela una sucesión de estados actuales de un sistema en el mundo físico, el campo de vectores captura las tendencias inherentes a muchas de esas trayectorias a comportarse de una 
manera determinada [...] [Estas] tendencias están representadas por singularidades en el campo de vectores, y como nota Deleuze, a pesar del hecho de que la naturaleza precisa de cada punto singular está bien definida solo en el retrato de fases (por la forma que las trayectorias toman en su vecindad), la existencia y la distribución de estas singularidades ya está completa en el campo de vectores (o direcciones)(DeLanda, 2002, p. 31).

Y así como el campo de vectores contiene la distribución entera de singularidades, incluyendo las singularidades no actualizadas todavía, así también en una multiplicidad deleuziana lo virtual es 'más-real', dado que contiene mayor información que muestra cómo un sistema podría actuar bajo condiciones todavía no actualizadas. El campo de vectores, si bien se distingue ontológicamente del retrato de fases, no debe ser tomado por una especie de reino trascendental donde se hallan las posibilidades; una mayor aclaración de las singularidades puede mostrar esto; por ejemplo, los atractores funcionan como puntos topológicos que 'guían' trayectorias específicas, pero dichas trayectorias siempre se aproximan a ellos de manera asintomática, i.e., se aproximan indefinidamente cerca sin alcanzarlos (DeLanda, 2002, p. 31). "Esto significa que, a diferencia de las trayectorias, las cuales representan estados actuales de objetos en el mundo, los atractores nunca son actualizados, dado que ningún punto de una trayectoria alcanza el atractor mismo [...]. Pero a pesar de su falta de actualidad, los atractores son sin embargo reales y tienen efectos definidos en entidades actuales" (DeLanda, 2002, pp. 31-32). Esto es lo que le permite a DeLanda definir más o menos cabalmente lo que es una multiplicidad para Deleuze: "Una multiplicidad es un conjunto anidado de campos de vectores relacionados entre sí por bifurcaciones simétrico-rompedoras, junto con la distribución de atractores que definen cada uno de sus niveles incrustados" (2002, pp. 31-32).

En resumen, la importancia del trasfondo técnico del concepto de multiplicidad es la siguiente. La variedad permite modelar procesos en espacios abstractos sin la necesidad de hacer referencia a un plano superior (más alto) que otorgue una unidad homogénea, y esta modelación se puede extender a sistemas físicos. Por su parte, la teoría de grupos permite relacionar procesos, a diferencia de propiedades, e introduce la noción clave de una transición simétrico-rompedora. Por último, el retrato de fases y el campo de vectores permiten representar la relación entre lo actual y lo virtual, lo que amplía el ámbito de lo que es concebido como real. En últimas, el concepto mismo de multiplicidad ofrece una manera de explicar de forma inmanente la morfogénesis. El trabajo de DeLanda va mucho más allá de lo que esta pequeña reconstrucción alcanza a capturar. DeLanda, luego de definir el concepto 
de multiplicidad y proponer una forma de pensar lo virtual, muestra cómo de allí puede surgir una explicación del espacio-tiempo extensivo de la actualidad con base en procesos diferenciadores intensivos. En efecto, DeLanda aquí está pensando en una recomendación que Deleuze y Guattari dejaron, según la cual: "es necesario retornar al interior de los estados o cuerpos de cosas científicos en el proceso de su constitución, para penetrar en la consistencia, esto es, en la esfera de lo virtual, una esfera que solo es actualizada allí. Sería necesario ascender por el camino por el cual la ciencia desciende, y al final del cual la lógica sitúa su campamento" (1994, p. 140). Para capturar la virtualidad habría que ir, no al objeto, sino a los procesos diferenciadores e intensivos mismos, donde se hace patente el plano de consistencia.

\section{IV}

¿Qué es lo que queda, entonces, después de haber discutido pájaros, mohos y átomos? Por ponerlo de manera abstracta, este descenso a modo de microscopio ha intentado mostrar que la expansión conceptual es productiva: la expresividad de Deleuze y Guattari muestra a Messiaen y sus pájaros-artistas como colaboradores; el moho creativo abre interrogantes sobre las posibilidades de sistemas no-centrados y lógicas computacionales alternativas; la ontología inmanentista y materialista invita a tomarse en serio la materia y sus procesos para pensar-en últimas-sobre cualquier cosa. Ahora el camino es hacia arriba. En un rápido ascenso habría que ver un mundo donde la materia, a través de procesos diferenciadores inmanentes a ella, se expresa (en tanto emite información) y crea (en tanto produce nuevas formas), convirtiéndose en el punto de partida para ver ya no el monopolio de la expresión (y el ejemplo puntual de creatividad) en la especie humana, sino un continuo que subyace a la materialidad misma. Así como Deleuze y Guattari ven el ritmo como un 'entredos', así también habría que ver el continuo de expresividad: pasos y traspasos entre lo subatómico y lo atómico, lo celular y lo molecular, lo inorgánico y lo orgánico, lo invertebrado y lo vertebrado, la planta y el animal. Ahora el moho creativo es también un pensador topológico, pues explora el espacio de posibilidades con sus tubos protoplásmicos y deja índices de su camino, mientras que los nutrientes que fungen como singularidades hacen que su tendencia a largo plazo sea la de hallar el camino más corto entre dos puntos, sin importar la distribución del espacio. Por último, habría que afirmar que el humano se halla convertido en un instante más de una larga serie de expresiones. En cualquier caso, aunque este artículo no sea más que un esbozo para buscar formas de desterritorializar la expresividad, sí es posible ver en él la formulación de un proyecto y las potencialidades de pensamiento que lo acompañan. 


\section{REFERENCIAS $^{3}$}

Adamatzky, A., \& Alonso-Sanz, R. (2011). Rebuilding Iberian motorways with slime mould. BioSystems, n. ${ }^{\circ} 105$,pp. 89-100.

Adamatzky, A., Armstrong, R., Jones, J., \& Gunji, Y. (2013). On creativity of slime mould. International Journal of General Systems, vol. 42, n. ${ }^{\circ}$, pp. 441457.

DeLanda, M. (1998). Deleuze and the Genesis of Form. Art Orbit, vol. 1, n. ${ }^{\circ}$, pp. 1-6.

DeLanda, M. (2002). Intensive Science and Virtual Philosophy. London: Continuum.

DeLanda, M. (2005). Material Expressivity. Domus, vol. 893, n. ${ }^{\circ}$, pp. 122-123.

Deleuze, G. (2002). Diferencia y repetición. (M. S. Delpy, Trad.) Buenos Aires: Amorrotu.

Deleuze, G. (1996). L'Abécédaire de Gilles Deleuze. Metropolis. (C. Parnet, Entrevistador).

Deleuze, G., \& Guattari, F. (2010). Mil mesetas. (J. V. Pérez, Trad.) Valencia: Pretextos.

Deleuze, G., \& Guattari, F. (1994). What is Philosophy? New York: Columbia University Press.

Grosz, E. (2008). Chaos, Territory, Art: Deleuze and the Framing of the Earth. New York: Columbia University Press.

Nakagaki, T. (2001). Smart behavior of true slime mold in a labyrinth. Res. Microbiol., n. ${ }^{\circ} 152$, pp. 767-770.

Nakagaki, T., Kobayashi, R., \& Tero, A. (2004). Physarum solver: A biologically inspired method of road-network navigation. Physica A, n. ${ }^{\circ} 363$, pp. 115119.

3 Las traducciones de los textos que están en inglés son de mi autoría. 
Nakagaki, T., Yamada, H., \& Hara, M. (2001). Smart network solutions in an amoeboid organism. Biophysical Chemistry, n. ${ }^{\circ}$ 107, pp. 1-5.

Nakagaki, T., Yamada, H., \& Tóth, A. (2001). Path finding by tube morphogenesis in an amoeboid organism. Biophysical Chemistry, n. ${ }^{\circ}$ 92, pp. 47-52.

Reid, C., Latty, T., Dussutour, A., \& Beekman, M. (2006). Slime mold uses an externalized spatial road-network navigation. Proceedings of the National Academy of Sciences, vol. 43, n. ${ }^{\circ} 109$, pp. 490-494.

Tsuda, S., Aono, M., \& Gunji, Y. (2004). Robust and emergent Physarum logicalcomputing. BioSystems, n. ${ }^{\circ} 73$, pp. 45-55. 